\title{
Modular Position-sensitive High-resolution Calorimeter for Use in Space Gamma-ray Instruments Based on Virtual Frisch-grid CdZnTe Detectors
}

\author{
Alexander Moiseev* \\ CRESST/NASA/GSFC and University of Maryland, College Park \\ College Park, MD 20772, USA \\ E-mail: alexander.a.moiseevenasa.gov
}

\section{Aleksey E. Bolotnikov}

Brookhaven National Laboratory

Upton, NY 11973, USA

\section{Carolyn Kierans}

NPP/NASA Goddard Space Flight Center

8800 Greenbelt Rd, Greenbelt, MD 20771, USA

\section{Elizabeth Hays, David J. Thompson}

NASA Goddard Space Flight Center

8800 Greenbelt Rd, Greenbelt, MD 20771, USA

\begin{abstract}
We present a calorimeter concept based on 3-dimensional (3D) position-sensitive virtual Frischgrid CdZnTe detectors. This calorimeter aims to measure photons with energies from $\sim 100 \mathrm{keV}$ to $20-50 \mathrm{MeV}$. The expected energy resolution at $662 \mathrm{keV}$ is better than $1 \%$ FWHM, and the photon interaction position-measurement accuracy is better than $1 \mathrm{~mm}$ in all dimensions. The 3D virtual voxel approach solves a long-standing problem with CdZnTe detectors stemming from material imperfections that limit the performance and usefulness of larger detectors. This concept enables relaxed requirements on the quality of crystals, while maintaining the same energy resolution, significantly reducing cost. A modular calorimeter design, consisting of rectangular prisms arranged in a grid, supports the assembly of large arrays. Such a detector can be used in gamma-ray telescopes that use Compton scattering, such as AMEGO, serving as a calorimeter and providing the position and energy measurement for scattered photons. The achievable energy resolution is suitable for spectroscopic measurements of gamma-ray lines from nuclear decays. In a combination with a coded aperture mask, the detector could also provide high angular resolution detection of gamma radiation.
\end{abstract}

36th International Cosmic Ray Conference -ICRC2019-

July 24th - August 1st, 2019

Madison, WI, U.S.A.

${ }^{*}$ Speaker. 


\section{Introduction}

The energy range from a few-hundred $\mathrm{keV}$ to a hundred $\mathrm{MeV}$ has remained mostly inaccessible since the Compton Gamma-Ray Observatory carried the COMPTEL instrument [1] in the 1990s. Future gamma-ray instruments, for example, the All-sky Medium-Energy Gamma-ray Observatory (AMEGO) [2], aim to explore the medium-energy gamma-ray band using a very large field-of-view and with a substantially increased sensitivity over COMPTEL, as well as enhanced angular resolution and energy resolution. The science goals for a medium-energy observatory (see for example, [3]) make use of the detection and characterization of transients and variable sources that emit the bulk of their emission in the $\mathrm{MeV}$ band, such as short gamma-ray burst (GRB) counterparts to binary neutron star mergers and gamma-ray blazars that peak in the $\mathrm{MeV}$ range. Medium-energy gamma-ray missions also seek to make spectroscopic measurements of electron-positron annihilation and nuclear line emission. These and other goals require an instrument that can detect gamma rays using both pair production and Compton scattering. In particular, observations between a few hundred $\mathrm{keV}$ and $\sim 10 \mathrm{MeV}$ demand detector technologies capable of making accurate position and energy measurements of both the scattered electron and photon from the Compton interaction. A position-sensitive, high-resolution CdZnTe calorimeter is being developed to address the need to absorb and measure the scattered Compton photon. In this submission we discuss the design and construction of a prototype CdZnTe calorimeter, present the preliminary performance characterization, and consider applications for this technology in a medium-energy observatory like AMEGO as well as in coded-mask telescope concepts.

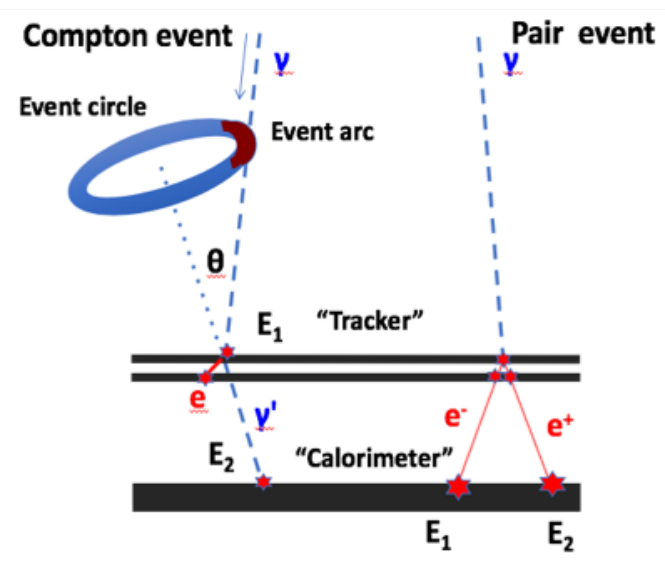

Figure 1: Photon interactions in the MeV range are dominated by Compton scattering at energies $\leq 10 \mathrm{MeV}$ (left) and pair-production at higher energies (right). An instrument bridging hundreds of keV to hundreds of $\mathrm{MeV}$ energies must use both of these techniques to detect and characterize the incoming photon, as shown. A calorimeter system measures the energy and position of the Compton-scattered photon and pair production products.

\section{Motivation for using virtual Frisch-grid CdZnTe detectors}

$\mathrm{CdZnTe}$ is a desirable material for detection of keV-MeV photons. It has a high atomic number, high density (important for gamma-ray detection efficiency), a low Fano-factor of 0.4, and 
good chemical and mechanical stability. Importantly, it can operate at room temperature with good energy resolution. CdZnTe detectors have been used in a number of space experiments already, e.g. Swift, InFocus, and NuStar. Despite the positive attributes, high-resolution energy measurements in large-volume detectors, such as those needed for the medium-energy gamma-ray band are challenging to achieve because of the effects of non-uniformities in CdZnTe crystals. Although crystal growing techniques have advanced substantially, higher-uniformity crystals are expensive and nonuniformities remain a challenge, setting a practical limit for the size of crystals that can be used for accurate position and energy measurements and the performance that can be achieved. This limitation reduces the feasibility for using these sensors in gamma-ray telescopes operating above hundreds of $\mathrm{keV}$ and up to hundreds of $\mathrm{MeV}$, where the light is not focused onto a focal plane detector, the cross section for interactions is low, and large volumes of crystal are necessary for adequate detection efficiencies and absorption of a Compton-scattered photon or pair-production products. For space telescope designs the ability to gather energy deposits over a large volume must also be balanced against minimizing the number of signal channels required to reduce the cost and complexity of the instrument.

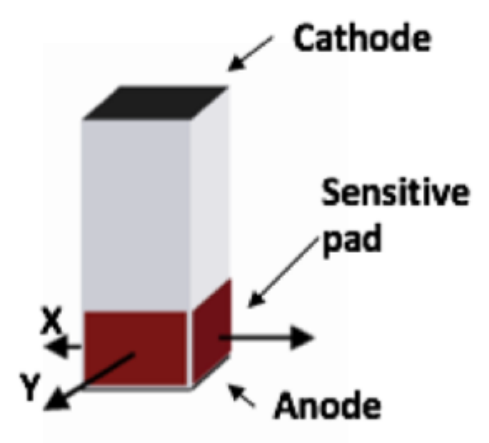

Figure 2: This cartoon depicts a CdZnTe bar configured to use the Frisch-grid technique to make 3D measurements of an interaction in the crystal. The anode and cathode signals give the drift time of the electron/hole pairs created after an interaction, and the $\mathrm{Z}$ coordinate of the interaction. The relative signals from the 4 side electrodes provide the $\mathrm{X}-\mathrm{Y}$ coordinates of the interaction.

The detector configuration developed by A. E. Bolotnikov [4] at Brookhaven National Laboratory for imaging and spectroscopy of gamma-ray sources using arrays of virtual Frisch-grid detectors provides a solution for the limitations of the crystal structure while achieving good position and energy resolution. The virtual Frisch-grid design employs a rectangular prism (bar) of CdZnTe with surrounding contact pads on the sides in addition to the cathode and anode on the ends (Fig 2). The combined use of the relative amplitude and timing of these signals compensates for effects of non-uniformities in the crystal, allowing the energy of the photon and the position of the interaction within the bar to be measured more accurately.

Along the longest dimension of the CdZnTe bar, defined as Z in Fig. 2, the variation in drift velocity affects the collected signal at the cathode and anode. This can be observed in the ratio of the cathode and anode signals, and conversely it gives a measure of the $\mathrm{Z}$ position of the interaction in the crystal. By measuring the $\mathrm{Z}$ position of the interaction using the relative signal strength in the cathode and anode, calibrations of the crystal allow corrections to be made that mitigate 
(a)

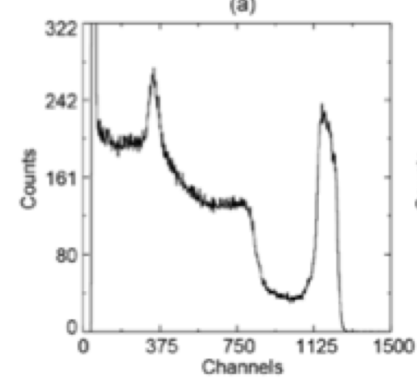

(b)

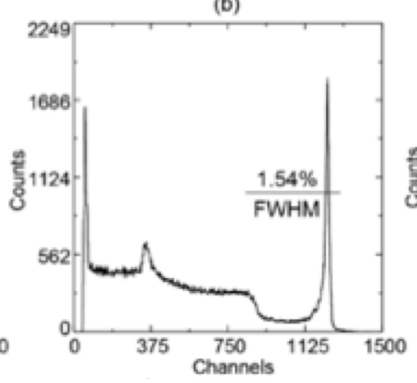

(c)

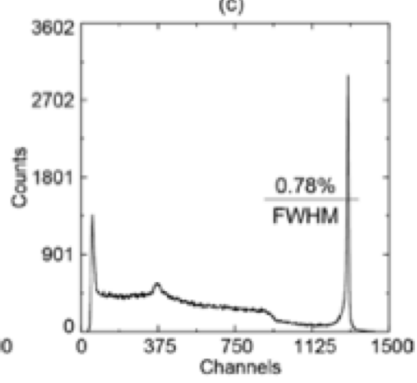

Figure 3: The spectral measurements at $662 \mathrm{keV}$ for a single CdZnTe bar demonstrate the improvement in energy resolution obtained using corrections for the interaction position within the crystal. Panel (a) shows the raw spectrum without any correction. The full width half maximum (FWHM) for the 662 line is $3 \%$. Panel (b) shows spectrum after including the 1D correction for the anode to cathode ratio, i.e. the $\mathrm{Z}$ position as defined in Fig. 2. The FWHM decreases to 1.54\%. Panel (c) shows the spectrum after adding the X-Y position to achieve a 3D correction using the signals from the crystal sides. The FWHM decreases to $0.78 \%$.

the effects caused by nonuniform sections of the crystal that lie between the interaction point and signal collection. The contacts distributed around the sides of the bar sample the signal in the $\mathrm{X}$ and $\mathrm{Y}$ dimensions. A comparison of the signals at these points provides the internal location of the interaction in the X-Y plane, which can be applied as a 2-dimensional correction in addition to the 1-dimensional $\mathrm{Z}$ correction. By measuring the full 3D position of the interaction with sufficient resolution, even low quality crystals can provide an accurate measurement of the energy deposited in the crystal (Fig. 3).

\section{Calorimeter Design}

The design of the prototype calorimeter employs several key features required for a larger instrument [5]. The overall structural approach is modular, with independent basic elements that can be arranged side by side, to allow scaling to large areas and because of the benefits for component testing and assembly.

The fundamental prototype calorimeter module consists of $16 \mathrm{CdZnTe}$ bars arranged in a $4 \times 4$ grid. Bars of size $6 \mathrm{~mm} \times 6 \mathrm{~mm} \times 20 \mathrm{~mm}$ and $8 \mathrm{~mm} \times 8 \mathrm{~mm} \times 20 \mathrm{~mm}$ were tested as part of this study. The bars must be held in an enclosure to protect them and to provide the distribution of electrical connections and bias voltage. The enclosure structure that has been selected (see Fig 4) works like an egg crate, encasing each bar in a separate cell.

The electronic digitization and application of the various sensor signals in correcting the measurements form a critical component of the detector system and are implemented in an ASIC. The signals must be sampled with suitable timing, polarity and dynamic range to achieve the potential of the spatial resolution of the interaction that allows for the spatial corrections to be applied to accurately reconstruct the deposited energy in the crystal. For the prototype an existing ASIC design was evaluated and adapted to improve the performance of the 16-bar array. An impact of wave-front sampling in comparison to more traditional shaped signal sampling used up to now for this device is shown in Fig. 5. 

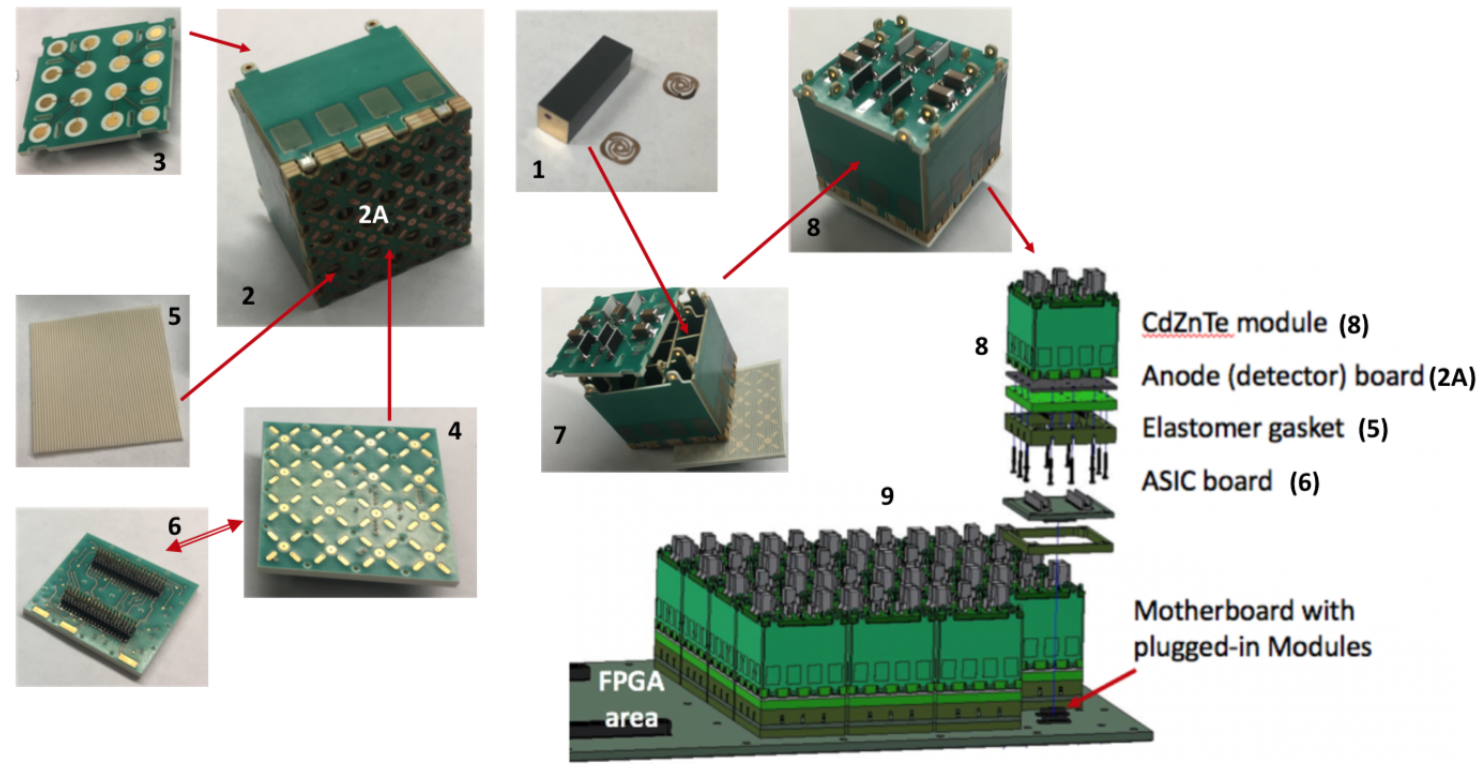

Figure 4: The prototype module assembly. 1: CdZnTe bar $(6 \mathrm{~mm} \times 6 \mathrm{~mm} \times 20 \mathrm{~mm})$ with gold-plated anode (cathode), and copper springs to be attached; 2: assembled module seen from the anode side; 3: cathode (HV) board shown from inside (the bars) side, with round pads to contact the cathode; 4: ASIC board, shown from inner (interface) side to be coupled with the module (2) through the elastomer gasket (5); 6: ASIC board, shown from the ASIC connector side. Can be changed according to the ASIC used; 7: the module from the cathode side, with cathode board slightly shifted to show the cells where the CZT bars are inserted; 8: fully assembled module, shown from the cathode side.

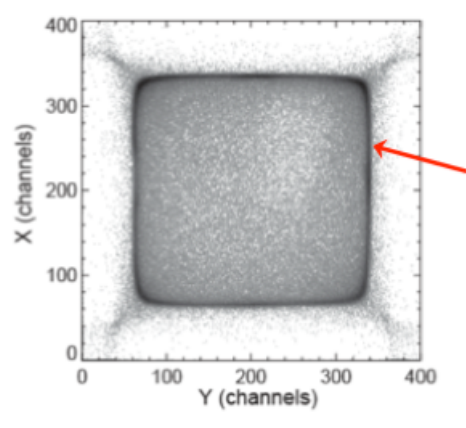

Using shaped signals (analog ASIC)
Artificial excess of events near the detector border due to poor sensitivity to the small signals

Much better with waveform digitization

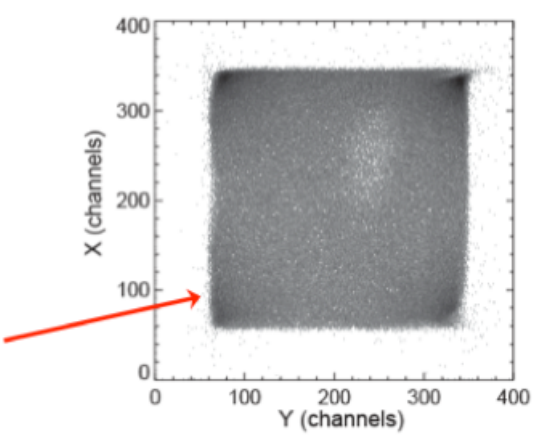

Using digitized waveforms

Figure 5: Reconstructed image of uniformly illuminated detector using an analog ASIC to process the signals (left) and by using discrete hybrid preamplifiers to digitize the waveforms (right). Distortion of the uniformity by the analog ASIC is visible and demonstrates the advantage of a wave-front sampling ASIC to realize the full performance potential of this technique. 


\section{Preliminary Performance Characterization}

To characterize the prototype modules, we conducted laboratory tests with various sources and beam tests using various the HIGS photon beam to demonstrate good energy resolution (Fig. 8) and efficiency of the developed 3D energy-correction method. Measurements of higher energy photons were limited to below $3 \mathrm{MeV}$ due to the dynamic range of the ASIC available for the initial testing.

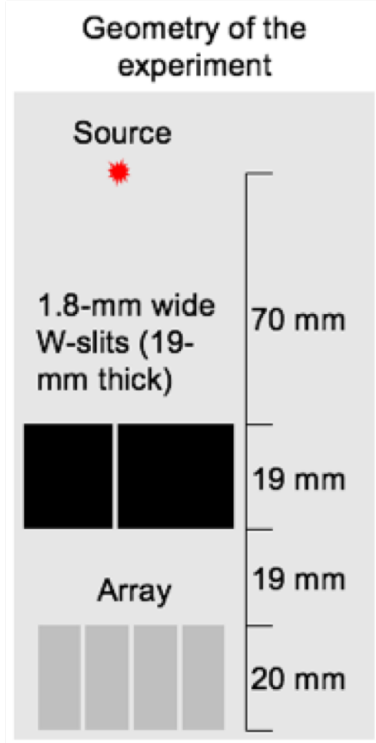

Figure 6: This diagram shows the physical configuration used for collimated slit tests conducted on the prototype array using various sources.

\section{Simulations}

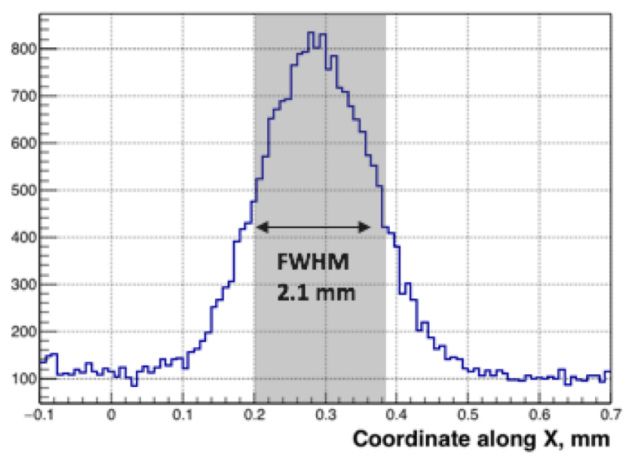

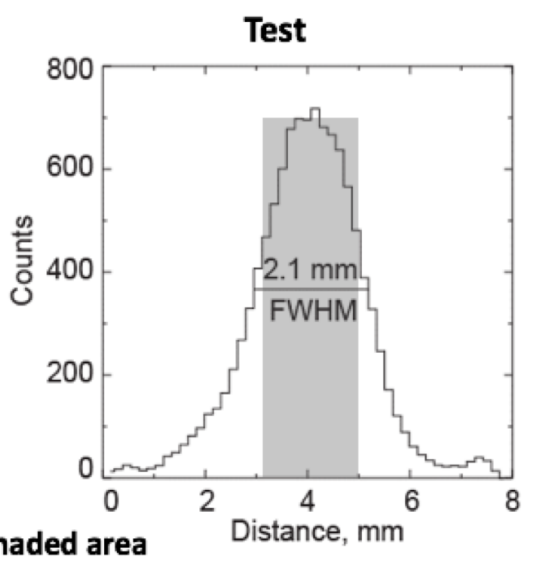

Figure 7: For the slit configuration shown in Fig. 6, the width of the measured signal observed in the 16-bar detector array (right) demonstrates a very close agreement with the expectations from simulations (left) of the device assuming a Gaussion with width $\sigma=0.5 \mathrm{~mm}$ for the spatial response. The shaded area represents the physical width of the slit used to collimate the beam.

In measurements of the position resolution performed using a collimated gamma-ray source (shown in Fig. 6), we observed visible distortion, especially near the detector edges (Fig. 5, left). 


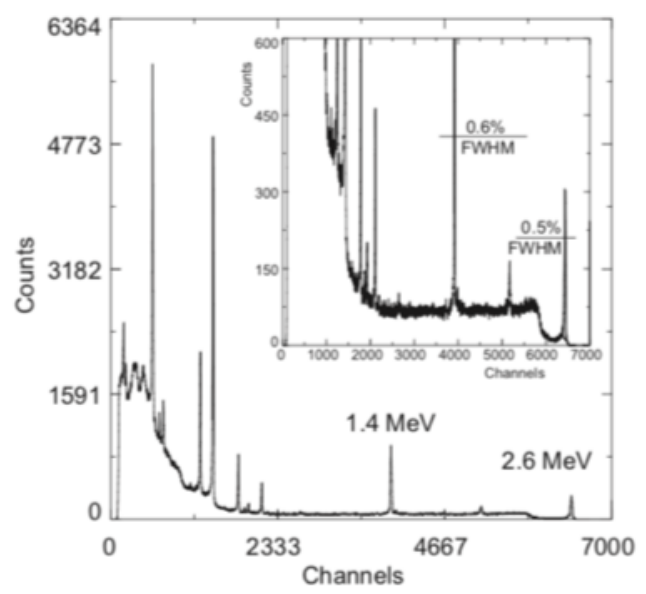

Figure 8: Spectrum measured from a U-232 source with gamma-ray lines up to $2.6 \mathrm{MeV}$ demonstrates high-energy resolution and high stopping power of thick CdZnTe detectors.

In our method, a point of energy deposition is reconstructed from a ratio of signals from sensitive pads and signals are measured by a conventional ASIC. Such an ASIC measures pulse-height with a certain shaping time. It is optimized for the expected signal polarity and works very well for measurements of signals from the anode and the cathode, which provide the energy measurement. The signals from the additional pads are bi-polar, and the signal polarity depends on where the energy deposition occurred with respect to the anode and the cathode. As a result, measurements show more prominent distortion for interactions that occur near the detector edge. The effect can be seen in Fig. 5 (left) as an artificial excess of events near the detector edge, in spite of the uniform illumination by the source. This effect can be mitigated by using a wave-front sampling ASIC (Fig. 5 , right.)

\section{Potential Applications}

The virtual Frisch-grid CdZnTe module was developed to improve direct imaging spectroscopy for gamma-ray sources, but this type of imaging has desirable applications in both direct and indirect applications in space telescopes. We have adapted that concept to explore improving the performance of a tracker and calorimeter pairing in a Compton-pair telescope design (Fig. 9, left), such as AMEGO, by taking advantage of the excellent spatial and energy resolution for the Compton-scattered photon or pair-production products. In AMEGO, an additional mode that selects direct detection of the initial Compton-scattering interaction in the CdZnTe calorimeter provides a significant enhancement to the effective area of the instrument (Fig. 9, middle). The possible applications of the technology are broad and also include use of such an array of modules as part of a high-angular-resolution focal plane imager for a coded-aperture telescope (Fig. 9, right; see also [6]). In all cases, the measurement precision at room temperatures and the potential to use larger-volume crystals at reduced quality provide a significant potential benefit for advancing medium-energy gamma-ray measurements. The preliminary beam test results presented here provide a start on studying feasible designs and performance for these applications for a device adapted to work in the space environment. 

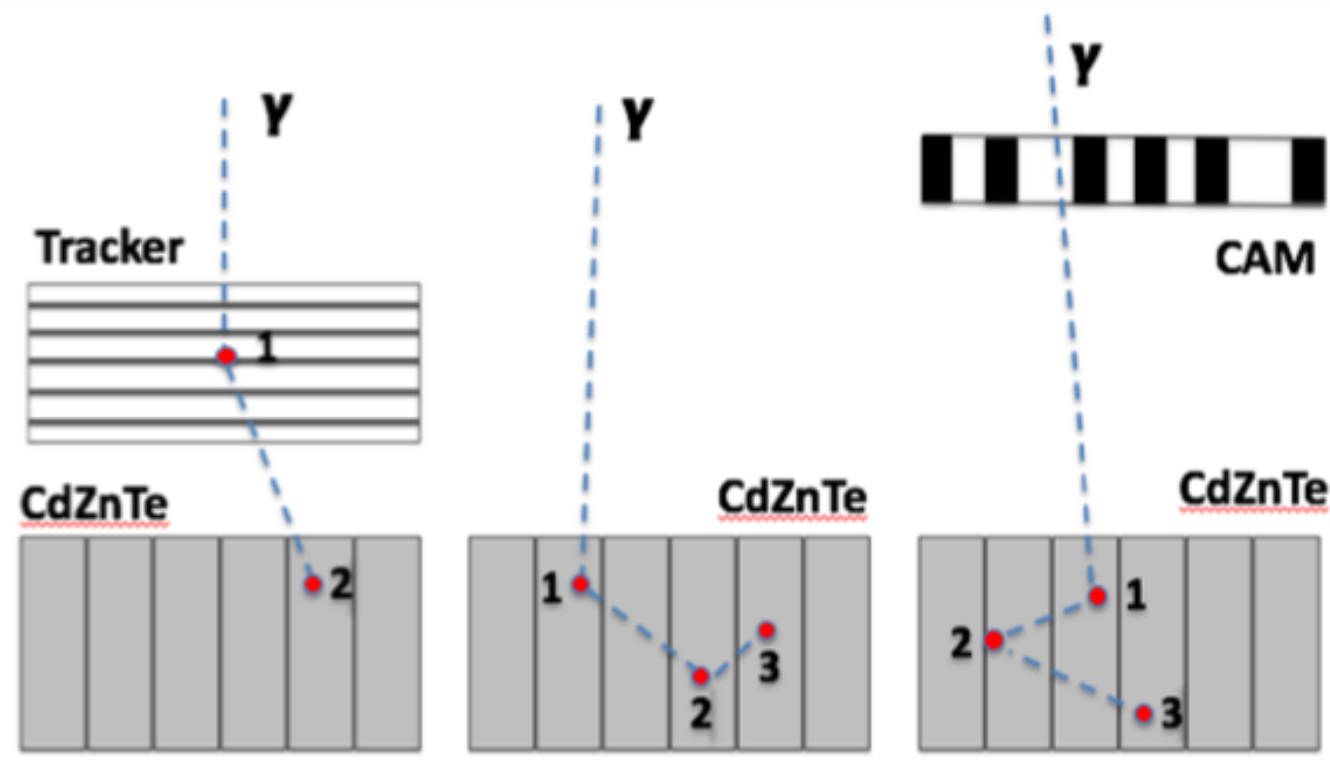

Figure 9: The diagrams represent three usage modes for the virtual Frisch-grid CdZnTe detectors. The left panel shows use in a 2-detector Compton telescope, where the Compton scatter happens in a separate tracking detector and the CdZnTe detects the scattered photon. The middle panel shows detection of a gamma-ray using multiple Compton scatters in the CdZnTe. The right panel shows use of the CdZnTe as the imaging detector for a coded-mask telescope.

\section{Summary}

We have constructed a single prototype module of a high spatial and energy resolution imaging calorimeter designed to be suitable for potential use in gamma-ray instruments. We find promising results from initial gamma-ray beam tests of a prototype consisting of $4 \times 4 \mathrm{CdZnTe}$ bars in the virtual Frisch-grid configuration and packaged to accommodate potential space applications. The energy resolution for this mode, measured at $0.78 \%$ at $662 \mathrm{keV}$ falls below the $1 \%$ goal for medium-energy gamma-ray instruments. Preliminary measurements of the resolution for the X-Y interaction location within the detector array at $\sim 0.5 \mathrm{~mm}$ for a collimated slit test suggest that the the goal of $<1 \mathrm{~mm}$ is also achievable. The next steps for advancing this work include further studies of wave-front sampling digitization of the signals from the side contacts for the CdZnTe bars. This mitigates the effect of bi-polar signals on the $\mathrm{X}-\mathrm{Y}$ position measurement. It will also be important to test this device using gamma-ray beams above $3 \mathrm{MeV}$, which are more likely to produce pair cascades and multiple interactions in a single crystal. Additional tests are being pursued to validate expectations for gamma-ray backgrounds, including inclusion with a balloon payload as part of the Compton-Pair Telescope Program, a prototype study for AMEGO.

\section{Acknowledgments}

The authors would like to acknowledge the support of NASA Astrophysics through the ROSES 
APRA program that funded the design, construction and initial tests of the prototype CZT module. We are grateful to Prof. Y. K. Wu and the High Intensity Gamma-Ray Source (HIGS) beam team at Duke University for providing the opportunity to run the tests presented in this work. We also wish to thank the Instrumentation Division and the Nonproliferation and National Security Department at Brookhaven National Laboratory for their aid in the effort to extend and adapt this technology to space-based gamma-ray telescopes.

\section{References}

[1] V. Schoenfelder et al, Instrument description and performance of the imaging gamma-ray telescope COMPTEL aboard the Compton Gamma-ray Observatory, ApJS 86:657-692, 1993.

[2] A. Moiseev et al., Compton-Pair Production Space Telescope for MeV Gamma-Ray Astronomy, arXiv:1508.07349 All-Sky Medium Energy Gamma-ray Observatory (AMEGO), in Proceedings of Science: 35th International Cosmic Ray Conference, Bexo, Busan, Korae, 2017.

[3] J. McEnery et al., All-sky Medium Energy Gamma-ray Observatory: Exploring the Extreme Multimessenger Universe, arXiv:1907.07558 Astro2020 APC White Paper, 2019.

[4] A. E. Bolotnikov et al., Use of high-granularity position sensing to correct response non-uniformities of CdZnTe detectors, Applied Physics Letters 104, 263503, 2014.

[5] A. Moiseev et al., High-Energy 3D Calorimeter based on position-sensitive virtual Frisch-grid CdZnTe detectors for use in Gamma-ray Astronomy, in Proceedings of Science: 35th International Cosmic Ray Conference, Bexo, Busan, Korae, 2017.

[6] A. Moiseev et al., New Mission Concept: Investigation of the Galactic Center with the Galactic Center Explorer (GalCenEx), in Proceedings of Science: 36th International Cosmic Ray Conference, Madison, WI, USA, 2019. 